\title{
The Molecular Envelopes of Planetary Nebulae
}

\author{
P. J. Huggins ${ }^{1}$, R. Bachiller ${ }^{2}$, P. Cox $^{3}$ and T. Forveille ${ }^{4}$ \\ ${ }^{1}$ New York University; ${ }^{2}$ OAN, Madrid; ${ }^{3}$ IAS, Univ. de Paris; ${ }^{4}$ Obs. de Grenoble
}

We report the results of an extensive survey of molecular gas in planetary nebulae (PNe) using the millimeter lines of $\mathrm{CO}$. The observations provide a range of information on the kinematics, the structure, and the mass of PN envelopes, which are important for understanding the formation and evolution of the ionized nebulae from the molecular envelopes ejected during the precursor AGB and proto-PNe phases.

The observations were made with the IRAM $30 \mathrm{~m}$ telescope at Pico Veleta (Spain) and the SEST $15 \mathrm{~m}$ telescope at La Silla (Chile) in the $1.3 \mathrm{~mm}(230 \mathrm{GHz}) \mathrm{CO}(J=2-1)$ line and the $2.6 \mathrm{~mm}(115 \mathrm{GHz}) \mathrm{CO}(J=1-0)$ line. The survey includes observations of 91 PNe: new detections or improved data for 23 objects in the $2-1$ and/or $1-0$ line, and sensitive upper limits for those not seen in $\mathrm{CO}$. The results are analyzed together with previous observations, and considerably extend the earlier survey work by Huggins \& Healy (1989). Detailed mapping of the structure and kinematics of individual objects is reported elsewhere (Bachiller et al. 1993, and references therein).

The observations confirm the existence of an important class of PNe with substantial envelopes of molecular gas. They include little known PNe (e.g., M1-13, He2-114, M3-3), as well as many familiar objects which have been well studied at other wavelengths (e.g., the Ring, the Dumbbell, the Helix, NGC 7027, NGC 2440). They range from compact to highly evolved $\mathrm{PNe}$, which implies that the molecular gas survives in the envelopes for a significant part of the evolution of the ionized nebulae. They also typically have abundance ratios of $\mathrm{N} / \mathrm{O}>0.3$ and elliptical or bipolar (with a waist) morphologies, indicating evolution from the more massive $\mathrm{PN}$ precursors of a young disk population.

Interpretation of the observed $\mathrm{CO}$ line intensities provides estimates for the $\mathrm{CO}$ column densities and the mass of molecular gas in the envelopes $\left(M_{m}\right)$. Our mass estimates assume that the $\mathrm{CO}$ molecules are fully associated and refer to the gas on the molecular side of the $\mathrm{CO} / \mathrm{C} \mathrm{I} / \mathrm{C}$ II transition at the ionized-neutral interface. An additional neutral mass component will be associated with the atomic gas in the transition regions but this has so far been measured in a only a few cases (see Bachiller et al. 1994, and references therein). The masses of the molecular envelopes estimated for the detected PNe are typically in the range $M_{m} \sim 10^{-2}$-a few $M_{\odot}$, and in more than a dozen cases they exceed the mass of the ionized nebula $\left(M_{i}\right)$ determined from the radio continuum emission. The column density through the envelopes and the mass ratio $M_{m} / M_{i}$ are shown in Fig. 1 versus the radius of the ionized nebulae $R$, which is roughly an indicator of the age of the PNe: the range $R \sim 0.003-0.3 \mathrm{pc}$ corresponds to expansion timescales of $\sim 100-10,000 \mathrm{yr}$. For the detected PNe, both the column density and the $M_{m} / M_{i}$ mass ratio show dramatic decreases with increasing nebular size, documenting the evolution of the envelopes as the ionized PNe form.

These PNe must evolve from AGB and proto-PN precursors that are surrounded by massive envelopes of molecular gas. As the central star rapidly evolves across the H-R diagram to higher temperatures, it becomes hot enough to begin ionizing the core of the 


\section{Envelopes}
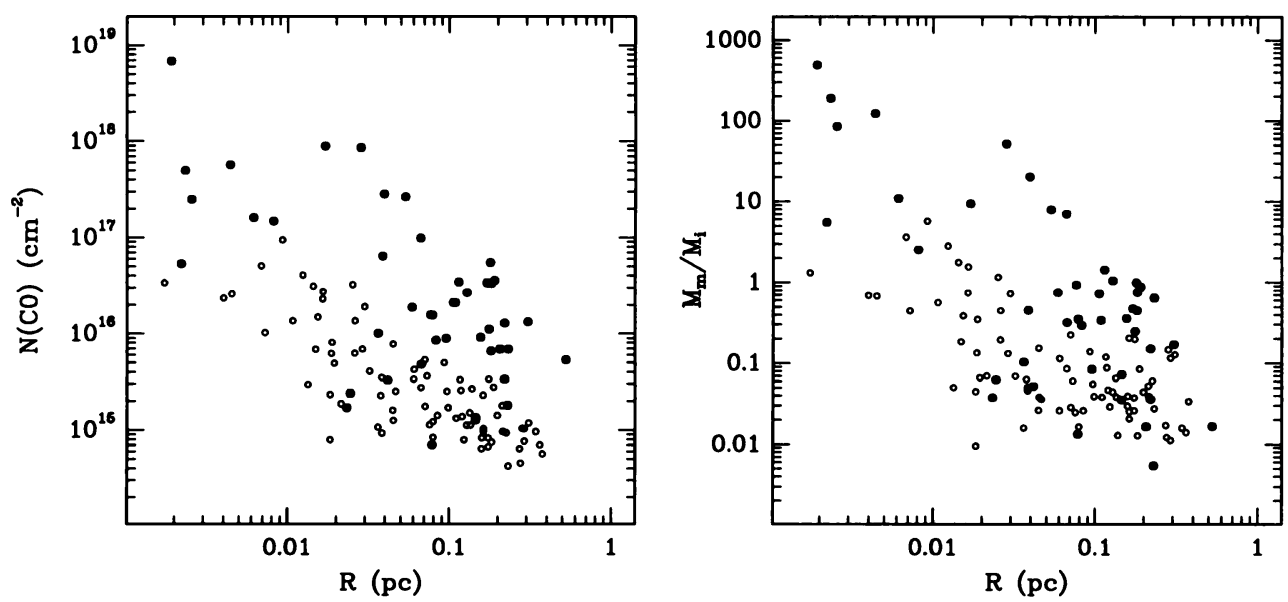

Figure 1: CO column density and molecular/ionized mass ratio vs. PN radius. Filled symbols are detected PNe, open symbols are upper limits.

expanding envelope, and forms a compact, ionization bounded PN, with $M_{m} / M_{i} \gg 1$. As the envelope continues to expand, the radial CO column density decreases, and the ionizing and dissociating stellar radiation consume more and more of the molecular gas. The ionized mass grows, and becomes comparable to the mass of molecular gas at a radius of $0.1-0.2 \mathrm{pc}$, and the major mass component at larger radii. High resolution molecular line images of these PNe, which show relatively complete envelopes for the compact objects and fragmented shells or isolated clumps in the more evolved PNe (e.g., Bachiller et al. 1993), strongly re-enforce this general scenario.

The nebulae not detected in $\mathrm{CO}$ have little or no molecular gas (typically $<10^{-2}$ $10^{-3} M_{\odot}$ ), and in many cases (see Fig. 1) their envelopes must be rapidly photo-dissociated before or during the compact phase. The large differences in the molecular gas content of the nebulae highlight the different evolutionary paths for planetary nebula formation which result from the range in mass of the progenitor stars and the structure of their circumstellar envelopes.

Further details can be found in Huggins et al. (1996). This work was supported in part by the National Science Foundation.

\section{REFERENCES}

Bachiller, R. Huggins, P. J. Cox, P., Forveille, 1993, Astron. Astrophys. 267, 177.

Bachiller, R. Huggins, P. J. Cox, P., Forveille, 1994, Astron. Astrophys. 281, L93.

Huggins, P. J., Healy, A. P., 1989, Astrophys. J., 346, 201.

Huggins, P. J., Bachiller, R., Cox, P., Forveille, T., 1996, Astron. Astrophys., in press. 